\title{
Ovarian Reserve in Women With Neuromyelitis Optica Spectrum Disorder
}

\author{
Jan Thöne ${ }^{1,2 *}$, Solveig Lichtenberg ${ }^{2}$, Anna Stahl ${ }^{3}$, Florence Pache ${ }^{4}$, Ingo Kleiter ${ }^{2}$, \\ Klemens Ruprecht ${ }^{4}$, Ralf Gold ${ }^{2}$ and Kerstin Hellwig ${ }^{2 *}$ \\ ${ }^{1}$ Department of Neurology, Katholische Kliniken Ruhrhalbinsel, Essen, Germany, ${ }^{2}$ Department of Neurology, St. Josef \\ Hospital, Ruhr-University Bochum, Bochum, Germany, ${ }^{3}$ Department of Pediatrics, Ruhr-University Bochum, Bochum, \\ Germany, ${ }^{4}$ Department of Neurology, Charité -Universitätsmedizin Berlin, Berlin, Germany
}

Neuromyelitis optica spectrum disorder (NMOSD) is a neuroinflammatory disease. The majority of NMOSD patients is seropositive for aquaporin-4 (AQP4) antibodies. AQP4 is the main water channel protein in the central nervous system, but has also been identified in the female reproductive system. Fertility issues and ovarian reserve has not yet been studied in females with NMOSD. The purpose of this study was to measure serum Anti-Müllerian hormone $(\mathrm{AMH})$ in females with NMOSD compared to healthy controls $(\mathrm{HC})$, in combination with other lifestyle and reproduction parameters. $\mathrm{AMH}$ is independent from the menstrual cycle and a reliable indicator of both ovarian reserve and ovarian function. We included a total of 32 reproductive-age females, $18 \mathrm{HC}$ and 14 with NMOSD. We used an enzymatically amplified two-site immunoassay to determine serum $\mathrm{AMH}$ level. In comparison to $\mathrm{HC}$, mean $\mathrm{AMH}$ value was reduced in NMOSD. Apart from that significantly more women with NMOSD showed low AMH levels $(<0.8 \mathrm{ng} / \mathrm{ml})$. Low $\mathrm{AMH}$ was associated with disease activity. In contrast, none of the immunotherapies for NMOSD, neither any reproductive life style parameter was associated with a decreased $\mathrm{AMH}$. Our results contribute to understanding of hindered fertility in females with NMOSD and enables neurologists to better counsel female patients.

Keywords: ovarian reserve, fecundity, childlessness, reproduction, autoimmune diseases of the nervous system

\section{INTRODUCTION}

Until detection of a highly specific serum immunoglobulin (Ig)-G autoantibody, neuromyelitis optica spectrum disorder (NMOSD) had previously been considered a multiple sclerosis (MS) variant. The diagnosis depends on recognizing the characteristic clinical picture, magnetic resonance imaging and detection of serum immunoglobulin (Ig)-G autoantibody (NMO-IgG) (1). The primary antigen for NMO-IgG is aquaporin-4 (AQP4), the main water channel protein in the central nervous system (CNS) (2). However, AQP4 has also been identified in a variety of other tissues including kidney, anterior pituitary, and in the reproductive system, to name just a few $(3,4)$.

Neuromyelitis optica spectrum disorder affects significantly more women than men by a ratio of 9-10/1 in seropositive and 2:1 in seronegative patients usually during their childbearing age, although symptoms can occur at any age from childhood to late adulthood $(2,5)$. 
Anti-Müllerian hormone (AMH), secreted by ovarian granulosa cells, is a homodimeric glycoprotein from the transforming growth factor-beta (TGF- $\beta$ ) superfamily (6). Accumulating evidence has shown that AMH is a good marker of ovarian reserve which describes the ovarian follicle quality and content at any given time. Usually, serum $\mathrm{AMH}$ levels in females peak during puberty, remain fairly constant during young adulthood but then bit by bit fall over reproductive time until it decreases below limit of detection at menopause $(7,8)$. AMH serum values show minimal diurnal or circadian variation and change during the menstrual cycle is low (9). This contrasts with variation in levels of other female sex hormones such as luteinizing hormone (LH), follicle stimulating hormone (FSH), estrogen and progesterone (10). We and others revealed that serum AMH levels are lower in reproductive aged women with immune mediated diseases such as MS and chronic inflammatory rheumatic diseases (10-12). We also found that AMH were lower in MS women not currently treated with immunomodulatory treatment. Furthermore, cytokines important in immune mediated disease may also affect ovarian function (13).

While the impact of pregnancy on NMOSD disease course has been evaluated, less reports assessed the influence of NMOSD on reproductive issues such as fertility, despite the fact that hypothamic lesions are common and therefore might contribute to fertility (14-16). In this study we analyzed serum AMH values in non-pregnant women with NMOSD. We also evaluated the potential contribution of disease modifying agents and disease activity on AMH.

\section{MATERIALS AND METHODS}

\section{Study Population}

Serum samples from reproductive-aged women with NMOSD $(n=14)$ were evaluated in a blinded fashion for the presence of serum AMH with a highly specific sandwich ELISA. Other covariates including demographics, body-mass index (BMI), hormonal contraception, age, and nicotine consumption were also collected. As age is the most important confounder for reduced ovarian reserve, we matched our cohort a priori for age. Age-matched healthy female controls (HC, $n=18)$ served as controls. Except oral contraception HC did not have other medications. All participants were of Caucasian origin and reported on a regular menstruation.

Exclusion criteria were: age $<18$ years and $>45$ years, pregnancy, current lactation, menopause, abnormal thyroid function, chronic liver or autoimmune disease (other than NMOSD), kidney or gynecological disease and therapy with agents toxic to reproduction (16). We also excluded females seeking advice on endocrine dysfunction and impaired fertility.

The protocol was approved by the institutional review board. Patients provided written informed consent before undergoing any procedures. All experiments have been carried out in accordance with The Code of Ethics of the World Association (Declaration of Helsinki). All datasets for this study are included in the manuscript and the Supplementary Files.

\section{Sample Collection and Enzyme Linked Immunosorbent Assay (ELISA) Experiments}

Serum were collected, aliquoted and stored at $-20^{\circ} \mathrm{C}$. Immediately before measurement, aliquots were brought to room temperature and analyzed for AMH using a sandwich ELISA kit (AMH Gen II ELISA, Beckman Coulter, Webster, USA) according to manufacturers' protocols.

\section{Statistical Analyses}

Statistical analyses were performed using the Prism software (GraphPad, San Diego, CA). Data are provided as mean \pm SEM. Significances between groups were examined using the MannWhitney $U$-test. Other statistical analyses were performed using repeated measurement ANOVA for multiple comparisons. In all experiments, a $p<0.05$ was defined as statistically significant and $p<0.01$ was considered as highly statistically significant.

\section{RESULTS}

\section{Clinical Data}

We included 14 women with NMOSD and 18 HCs. Characteristics of NMOSD patients are summarized in Table $\mathbf{1 .}$ Groups did not differ significantly with respect to age, BMI, use of birth control pill and smoking habits (Supplementary Table 1).

\section{Reproductive and Gynecological History}

The age at menarche did not differ between groups and only 3 participants reported on an irregular menstrual period (Supplementary Table 1). In the NMOSD group, 3 patients (21.4\%) had a total of 6 children compared to 3 HCs (16.6\%) with a total of 5 children. With one exception females remembered the interval after contraception has been discontinued and the validation of pregnancy. The weeks to pregnancy did not differ between both groups (Supplementary Table 1). No woman had a history of assisted reproductive intervention or miscarriages.

\section{NMOSD Disease Specific Characteristics}

Median EDSS was 3.5 (range 1.0-7.5). Median disease duration was six years (range 1-10). All females with NMOSD were currently treated. The majority received either rituximab $(n=5$, $35.7 \%)$ or azathioprine $(n=5,35.7 \%)$ followed by tocilizumab ( $n$ $=2 ; 28.6 \%)$, teriflunomide $(n=1,7.1 \%)$ and dimethyl fumarate $(n=1 ; 7.1 \%)$. Antibodies to AQP4 were found in eleven women (78.6\%) with NMOSD. One AQP4-IgG-seronegative NMOSD patient (7.1\%) was seropositive for myelin oligodendrocyte glycoprotein antibodies (MOG-IgG). Two NMOSD patients (14.2\%) were seronegative for both AQP4-IgG and MOG-IgG. No other antibodies were reported in all NMOSD patients.

\section{Serum AMH}

The mean AMH level was $2.125 \pm 0.47 \mathrm{ng} / \mathrm{ml}$ in the NMOSD group compared to $3.250 \pm 0.48 \mathrm{ng} / \mathrm{ml}$ in HCs (Figure 1, Supplementary Table 2). Next, AMH was categorized in biological meaningful categories $(<0.8 \mathrm{ng} / \mathrm{ml}=$ low $\mathrm{AMH}$; $<0.4 \mathrm{ng} / \mathrm{ml}=$ very low AMH). Significantly more females with NMOSD $(n=4,28.6 \%)$ had AMH levels $<0.8 \mathrm{ng} / \mathrm{ml}$ 
TABLE 1 | Characteristics of NMOSD patients (\#1-14) and HC (\#15-33).

\begin{tabular}{|c|c|c|c|c|c|c|c|c|c|c|}
\hline$\#$ & Age & Onset & EDSS & No. of relapses & Current medication & $A Q P 4(x) \lg G$ & MOG- IgG & AMH ng/ml & Birth control & Children \\
\hline 1 & $25-30$ & 2006 & 7 & 29 & Tocilizumab & + & - & 0.457 & - & - \\
\hline 2 & $40-45$ & 2006 & 6 & 2 & Teriflunomide & + & - & 6.543 & - & - \\
\hline 3 & $40-45$ & 2010 & 7.5 & 9 & Tocilizumab & + & - & 0.827 & - & - \\
\hline 4 & $35-40$ & 2008 & 5 & 10 & Azathioprin & - & - & 0.211 & - & - \\
\hline 5 & 30-35 & 2007 & 2.5 & 11 & Rituximab & + & - & 0.765 & + & - \\
\hline 6 & $35-40$ & 2013 & 4 & 8 & DMF & - & - & 2.928 & + & + \\
\hline 7 & $20-25$ & 2014 & 1.5 & 3 & Rituximab & - & + & 1.821 & + & - \\
\hline 8 & $20-25$ & 2008 & 1.5 & 2 & Azathioprin & + & - & 3.538 & + & + \\
\hline 9 & $20-25$ & 2015 & 2 & 2 & Rituximab & + & - & 1.456 & + & - \\
\hline 10 & 30-35 & 2011 & 2.5 & 1 & Rituximab & + & - & 1.335 & - & - \\
\hline 11 & $15-20$ & 2010 & 3.5 & 5 & Azathioprin & + & - & 4.235 & - & - \\
\hline 12 & $40-45$ & 2014 & 1 & 1 & Rituximab & + & - & 0.511 & - & - \\
\hline 13 & $40-45$ & 2010 & 1 & 8 & Azathioprin & + & - & 2.187 & - & + \\
\hline 14 & $35-40$ & 2009 & 2.5 & 4 & Azathioprin & + & - & 2.940 & - & - \\
\hline 15 & $30-35$ & $x$ & $x$ & $x$ & None & $x$ & $x$ & 3.779 & + & + \\
\hline 16 & $40-45$ & $x$ & $x$ & $x$ & None & $x$ & $x$ & 1.191 & - & - \\
\hline 17 & $40-45$ & $x$ & $x$ & $x$ & None & $x$ & $x$ & 0.892 & - & + \\
\hline 18 & $35-40$ & $x$ & $x$ & $x$ & None & $x$ & $x$ & 0.648 & - & - \\
\hline 19 & $30-35$ & $x$ & $x$ & $x$ & None & $x$ & $x$ & 1.875 & - & - \\
\hline 20 & $40-45$ & $x$ & $x$ & $x$ & None & $x$ & $x$ & 0.909 & - & - \\
\hline 21 & $20-25$ & $x$ & $x$ & $x$ & None & $x$ & $x$ & 4.936 & + & - \\
\hline 22 & $20-25$ & $x$ & $x$ & $x$ & None & $x$ & $x$ & 4.901 & + & - \\
\hline 23 & $30-35$ & $x$ & $x$ & $x$ & None & $x$ & $x$ & 4.304 & - & - \\
\hline 24 & 30-35 & $x$ & $x$ & $x$ & None & $x$ & $x$ & 6.394 & - & - \\
\hline 25 & $20-25$ & $x$ & $x$ & $x$ & None & $x$ & $x$ & 3.922 & + & - \\
\hline 26 & 30-35 & $x$ & $x$ & $x$ & None & $x$ & $x$ & 1.496 & + & - \\
\hline 27 & $30-35$ & $x$ & $x$ & $x$ & None & $x$ & $x$ & 2.418 & - & - \\
\hline 28 & $40-45$ & $x$ & $x$ & $x$ & None & $x$ & $x$ & 0.929 & - & - \\
\hline 29 & $25-30$ & $x$ & $x$ & $x$ & None & $x$ & $x$ & 7.057 & + & - \\
\hline 30 & 30-35 & $x$ & $x$ & $x$ & None & $x$ & $x$ & 5.623 & + & + \\
\hline 31 & $20-25$ & $x$ & $x$ & $x$ & None & $x$ & $x$ & 2.461 & + & - \\
\hline 32 & $25-30$ & $x$ & $x$ & $x$ & None & $x$ & $x$ & 4.757 & + & - \\
\hline
\end{tabular}

In order to protect patients identify and in accordance with journal regulations the patient's age is presented as a range. Dimethyl fumarate (DMF).

in comparison to $\mathrm{HC}(n=1,5.5 \%)$. We observed very low $\mathrm{AMH}$ values only in one NMOSD patient (7.1\%) (Table 1). All reproductive life style factors were not associated with an $\mathrm{AMH}$ level $<0.8 \mathrm{ng} / \mathrm{ml}$ in the bivariate analysis, neither were NMOSD immune treatments. There was a non-significant difference in EDSS severity between women with low and normal $\mathrm{AMH}$ (EDSS: $4.1 \pm 1.1$ vs. $3.2 \pm 0.6 ; p=0.472$ ). Additionally, females with low AMH reported on more relapses compared to females with normal AMH $(12.75 \pm 5.8$ vs. $5.1 \pm 1.0 ; p=0.193)$. Neither disease duration (years: $5.0 \pm 2.3$ vs. $4.3 \pm 2.4$ ) nor age at sample collection ( $35.2 \pm 5.6$ vs. $32.4 \pm 9.6)$ differed significantly between females with low $\mathrm{AMH}$ and normal $\mathrm{AMH}$.

\section{DISCUSSION}

This is the first case series showing that women with NMOSD have lower mean /median AMH levels than healthy controls.
We also noted that biological meaningful low levels of $\mathrm{AMH}$ $(<0.8 \mathrm{ng} / \mathrm{ml})$ were significantly more frequent in women with NMOSD, consistent with recent studies in females with MS $(10,11)$. Low AMH was not associated with treatment or other investigated factors.

Our data indicate that ovarian reserve may be impaired in a fraction of female NMOSD patients although the biological reasons remain yet questionable.

An increasing number of women delay childbearing and often numerous years of contraception may precede the plan to conceive. But the size of ovarian reserve and consequently fertility declines throughout life (17). Whereas 1 in 10 women has trouble becoming pregnant $50 \%$ of women aged $40-44$ report on difficulties becoming pregnant (18); Centers for disease control and prevention, $2013^{1}$. In our case series patients were

\footnotetext{
${ }_{1}^{1}$ https://www.cdc.gov/nchs/fastats/infertility.htm (Accessed July 8, 2013).
} 


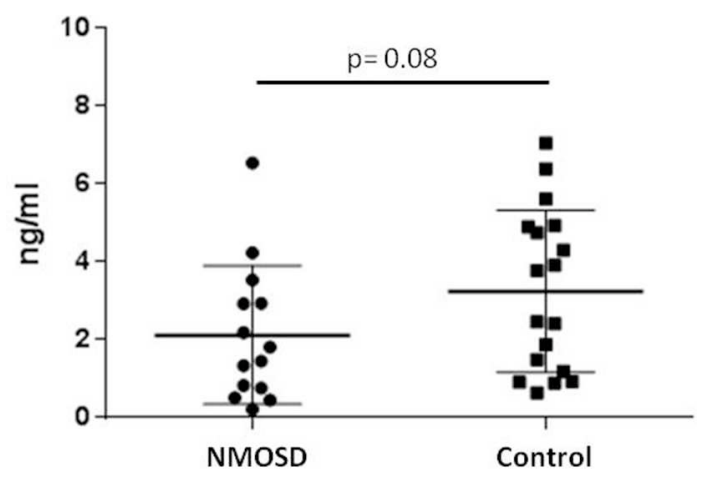

FIGURE 1 | Anti-Müllerian Hormone (AMH) ELISA: NMOSD subjects show reduced mean $\mathrm{AMH}$ serum level compared to healthy controls (Mann-Whitney U-Test).

age matched and therefore age differences as the strongest confounder for a reduced ovarian reserve cannot explain our results. Apart from that women have to face potential long-term side effects of immunotherapies, including potential unfavorable effects on fertility, and fecundity.

The cause and significance of AMH decrease in NMOSD females remains yet unclear. Ovulation is a complex process on a monthly basis orchestrated by a balanced interaction between various sex steroids. This process can be disturbed both on a local immunological aberrations and on a neuroendocrine level.

AQP4 has been identified in different tissues outside of the CNS but was not found in the human ovary including stroma, cortex and follicles, suggesting that NMO-IgG dependent inflammatory processes of the ovaries and following diminished ovarian reserve is implausible (3). Thyroid function was inconspicuous in all participants. Finally, neither certain NMOSD therapies nor any reproductive lifestyle parameter was associated with low AMH. Similar findings were reported in women with MS $(10,11)$.

Taken these aspects into account an impairment of the hypothalamic-pituitary-ovary axis (HPOA) seems to be the most attractive cause of AMH decrease and poorer ovarian reserve. Previous studies in AQP4 knockout mice demonstrated disrupted secretion of hormones from the HPOA and subfertility $(4,19)$. Interestingly, before puberty the appearance of ovaries of AQP4 knockout mice was similar to control mice, but at maturation, the ovaries of knockout mice revealed a marked reduction in follicular maturation (4).

The mammalian ovary has a defined supply of oocytes. Female that enter puberty with a diminished number of oocytes are susceptible of a faster reproductive decline, as their ovarian follicle content is expected to decline faster. Poorer ovarian reserve does not necessarily compromise fecundity and here, pregnancy rates did not differ between groups. Nevertheless, poor ovarian reserve may lead to a premature menopause and subsequent risk of childlessness. Indeed, $\mathrm{AMH}$ knockout mice are characterized by a faster depletion of their primordial follicle pool compared to control animals (20). Furthermore, very recently (21) reported on a decreased number of pregnancies and a notable rate of fertility treatments in women with NMOSD (21).

Our study is limited by the small sample size, the etiologic heterogeneity of the NMOSD patients included here and the cross-sectional design, hence, we cannot establish an actual temporal sequence to prove causality. Another potential drawback is the exclusion of known comorbidities and the inclusion of patients on immunotherapies that may both influence fertility as well. Therefore, we can not survey the real effect of NMOSD on reproductive history. One strength of our study is that this is, to our knowledge, the first examination of $\mathrm{AMH}$ in NMOSD females as a marker of ovarian reserve and the classification of $\mathrm{AMH}$ in biological significant categories. Our data suggest that females with NMOSD evidence might show a modified endocrine pattern and provide the motivation for future research. Additionally, our data enables neurologists to better advise women with NMOSD with the plan to conceive.

In conclusion, we have shown for the first time that in women with NMOSD serum AMH, an indirect marker of ovarian reserve, is reduced compared to healthy controls. Our data reveal further evidence of a modified endocrine pattern in some women with NMOSD and provide the rationale for future studies.

\section{ETHICS STATEMENT}

All participants gave written informed consent. The study protocol was approved by the Institutional Ethical Committee of the Ruhr University Bochum, Germany.

\section{AUTHOR CONTRIBUTIONS}

JT devised research, collected data, performed the statistical analysis, and wrote the manuscript. SL, FP, and AS collected data. $\mathrm{KH}$ conceived study, designed research, analyzed data, and wrote sections of the manuscript. IK conceived study, designed research, and wrote sections of the manuscript. RG and KR conceived study, designed research. All authors contributed to manuscript revision, read and approved the submitted version.

\section{ACKNOWLEDGMENTS}

The questionnaire about gynecological and obstetric history was kindly provided by Barbara Lawrenz, University Hospital for Women, Tuebingen, Germany.

\section{SUPPLEMENTARY MATERIAL}

The Supplementary Material for this article can be found online at: https://www.frontiersin.org/articles/10.3389/fneur. 2018.00446/full\#supplementary-material 


\section{REFERENCES}

1. Wingerchuk DM, Banwell B, Bennett JL, Cabre P, Carroll W, Chitnis T, et al. International consensus diagnostic criteria for neuromyelitis optica spectrum disorders. Neurology (2015) 85:177-89. doi: 10.1212/WNL.0000000000001729

2. Jarius S, Wildemann B, Paul F. Neuromyelitis optica: clinical features, immunopathogenesis and treatment. Clin Exp Immunol. (2014) 176:149-64. doi: $10.1111 /$ cei.12271

3. Saadoun S, Waters P, Leite MI, Bennett JL, Vincent A, Papadopoulos MC, Neuromyelitis optica IgG causes placental inflammation and fetal death. $J$ Immunol. (2013) 191:2999-3005. doi: 10.4049/jimmunol.1301483

4. Sun XL, Zhang J, Fan Y, Ding JH, Sha JH, Hu G. Aquaporin-4 deficiency induces subfertility in female mice. Fertil Steril. (2009) 92:1736-43. doi: 10.1016/j.fertnstert.2008.07.1785

5. Pandit L, Asgari N, Apiwattanakul M, Palace J, Paul F, Leite MI, et al. GJCF International Clinical Consortium \& Biorepository for Neuromyelitis Optica. Demographic and clinical features of neuromyelitis optica: a review. Mult Scler. (2015) 21, 845-53. doi: 10.1177/1352458515572406

6. Visser JA, Schipper I, Laven JS, Themmen AP. Anti-Müllerian hormone: an ovarian reserve marker in primary ovarian insufficiency. Nat Rev Endocrinol. (2012) 8:331-41. doi: 10.1038/nrendo.2011.224

7. Kelsey TW, Wright P, Nelson SM, Anderson RA, Wallace WH. A validated model of serum anti-müllerian hormone from conception to menopause. PLoS ONE (2011) 6:e22024. doi: 10.1371/journal.pone.0022024

8. Seifer DB, Baker VL, Leader B. Age-specific serum anti-Müllerian hormone values for 17,120 women presenting to fertility centers within the United States. Fertil Steril. (2011) 95, 747-50. doi: 10.1016/j.fertnstert.2010.10.011

9. La Marca A, Grisendi V, Griesinger G. How much does AMH really vary in normal women? Int J Endocrinol. (2013) 2013:959487. doi: $10.1155 / 2013 / 959487$

10. Thöne J, Kollar S, Nousome D, Ellrichmann G, Kleiter I, Gold R, et al. Serum anti-Müllerian hormone levels in reproductive-age women with relapsing-remitting multiple sclerosis. Mult Scler. (2015) 21:41-7. doi: $10.1177 / 1352458514540843$

11. Sepúlveda M, Ros C, Martínez-Lapiscina EH, Solà-Valls N, Hervàs $M$, Llufriu S, et al. Pituitary-ovary axis and ovarian reserve in fertile women with multiple sclerosis: a pilot study. Mult Scler. (2016) 22:564-8. doi: $10.1177 / 1352458515602339$

12. Henes M, Froeschlin J, Taran FA, Brucker S, Rall KK, Xenitidis T, et al. Ovarian reserve alterations in premenopausal women with chronic inflammatory rheumatic diseases: impact of rheumatoid arthritis, Behçet's disease and spondyloarthritis on anti-Müllerian hormone levels. Rheumatology (2015) 54:1709-12. doi: 10.1093/rheumatology/kev124

13. Paradisi R, Vicenti R, Macciocca M, Seracchioli R, Rossi S, Fabbri R. High cytokine expression and reduced ovarian reserve in patients with Hodgkin lymphoma or non-Hodgkin lymphoma. Fertil Steril. (2016) 106:1176-82. doi: 10.1016/j.fertnstert.2016.06.035

14. Bourre B, Marignier R, Zéphir H, Papeix C, Brassat D, Castelnovo G, et al. Neuromyelitis optica and pregnancy. Neurology (2012) 78:875-9. doi: 10.1212/WNL.0b013e31824c466f

15. Nour MM, Nakashima I, Coutinho E, Woodhall M, Sousa F, Revis J. et al. Pregnancy outcomes in aquaporin-4-positive neuromyelitis optica spectrum disorder. Neurology (2016) 86:79-87. doi: 10.1212/WNL.0000000000002208
16. Shosha E, Pittock SJ, Flanagan E, Weinshenker BG. Neuromyelitis optica spectrum disorders and pregnancy: Interactions and management. Mult Scler. (2017) 23:1808-17. doi: 10.1177/1352458517740215

17. Borisow N, Kleiter I, Gahlen A, Fischer K, Wernecke KD, Pache F, et al. Influence of female sex and fertile age on neuromyelitis optica spectrum disorders. Mult Scler. (2017) 23:1092-103. doi: 10.1177/1352458516671203

18. Gnoth C, Godehardt D, Godehardt E, Frank-Herrmann P, Freundl G. Time to pregnancy: results of the German prospective study and impact on the management of infertility. Hum Reprod. (2003) 18:1959-66. doi: 10.1093/humrep/deg366

19. Sun XL, Ding JH, Fan Y, Zhang J, Gao L, Hu G. Aquaporin 4 regulates the effects of ovarian hormones on monoamine neurotransmission. Biochem Biophys Res Commun. (2007) 353:457-62. doi: 10.1016/j.bbrc.2006. 12.040

20. Durlinger AL, Visser JA, Themmen AP. Regulation of ovarian function: the role of anti-Müllerian hormone. Reproduction (2002) 124:601-9. doi: 10.1530/rep.0.1240601

21. Bove R, Elsone L, Alvarez E, Borisow N, Cortez MM, Mateen FJ, et al. Female hormonal exposures and neuromyelitis optica symptom onset in a multicenter study. Neurol Neuroimmunol Neuroinflamm. (2017) 4:e339. doi: 10.1212/NXI.0000000000000339

Conflict of Interest Statement: KH, IK, and KR are member of a German-wide network of NMO researchers and clinical neurologists; Neuromyelitis optica study group (NEMOS). KH is supported by a research grant of the German Research Foundation (He 6841/1-1) and has received speaker and research Honoria from Bayer Healthcare, Biogen Idec, Teva Pharmaceutical Industries Ltd, Merck Serono, Sanofi Aventis and Novartis Pharma. IK received honoraria for consultancy or speaking and travel reimbursement from Bayer Healthcare, Biogen, Chugai, Merck, Roche, and Shire, and research support from Affectis, Biogen, Chugai and Diamed, all not related to the presented work. RG reported serving on scientific advisory boards for Teva Pharmaceutical Industries Ltd, Biogen Idec, Bayer Schering Pharma, and Novartis; receiving speaker honoraria from Biogen Idec, Teva Pharmaceutical Industries Ltd, Bayer Schering Pharma, and Novartis; and receiving research support from Teva Pharmaceutical Industries Ltd, Biogen Idec, Bayer Schering Pharma, Merck Serono, and Novartis. KR received grants from German Ministry of Education and Research (BMBF/KKNMS, Competence Network Multiple Sclerosis), Novartis, Merck Serono and the Charite Research Fund; honoraria for consultancy or speaking and travel reimbursement from Novartis, Bayer Healthcare, Biogen Idec, Merck Serono, sanofi-aventis/Genzyme, Teva Pharmaceuticals, and Guthy Jackson Charitable Foundation; all unrelated to the submitted work.

The remaining authors declare that the research was conducted in the absence of any commercial or financial relationships that could be construed as a potential conflict of interest.

Copyright (๑) 2018 Thöne, Lichtenberg, Stahl, Pache, Kleiter, Ruprecht, Gold and Hellwig. This is an open-access article distributed under the terms of the Creative Commons Attribution License (CC BY). The use, distribution or reproduction in other forums is permitted, provided the original author(s) and the copyright owner are credited and that the original publication in this journal is cited, in accordance with accepted academic practice. No use, distribution or reproduction is permitted which does not comply with these terms. 